\title{
Seamless Bit-Stream Switching in Multirate-Based Video Streaming Systems
}

\author{
Wei Zhang and Bing Zeng \\ Department of Electrical and Electronic Engineering, The Hong Kong University of Science and Technology, Clear Water Bay, \\ Kowloon, Hong Kong
}

Received 15 August 2005; Revised 18 December 2005; Accepted 15 March 2006

\begin{abstract}
This paper presents an efficient switching method among non-scalable bit-streams in a multirate-based video streaming system. This method not only takes advantage of the high coding efficiency of non-scalable coding schemes (compared with scalable ones), but also allows a high flexibility in streaming services to accommodate the heterogeneity of real-world networks. One unique feature of our method is that, at every preselected switching point, the reconstructed frame at each rate or two reconstructed frames at different rates will go through an independent or a joint processing in the wavelet domain, using an SPIHT-type coding algorithm. Another important step in our method is that we will apply a novel bit allocation strategy over all hierarchical trees that are generated after the wavelet decomposition so as to achieve a significantly improved coding quality. Compared with other existing methods, our method can achieve the seamless switching at each preselected switching point with a better rate-distortion performance.
\end{abstract}

Copyright ( 2006 Hindawi Publishing Corporation. All rights reserved.

\section{INTRODUCTION}

Due to the rapid growth and wide coverage of the Internet in recent years, there is a great increase of demand on various video services over the Internet, especially the real-time video streaming service. In contrast with the download mode where a video session is downloaded entirely to a user before it can be played, real-time video streaming enables users to enjoy the video service right after a very small portion of the whole video session is received. However, the Internet is an inherently heterogeneous and dynamic network, that is, the connecting bandwidth between the server and each user is varying with time. Under such circumstance of varying bandwidth, how to maintain a robust quality of service (QoS) is perhaps the most challenging requirement during each service session. In response to this challenge, two different source coding approaches have been developed in recent years, which are briefly outlined in the following.

\subsection{Multirate non-scalable coding scheme versus scalable coding scheme}

One straightforward solution to the challenge mentioned above is to perform a multiple bit-rate (MBR) representation, that is, to encode each source video into multiple nonscalable bit-streams, each at a preselected bit-rate. At each time-slot during the streaming service, an appropriate bitstream is selected according to the available bandwidth and then transmitted to the user. Clearly, each bit-stream generated here can be encoded optimally at the chosen bit-rate. On the other hand, however, it is also clear that we cannot make the best use of the available bandwidth when it is between two preselected rates.

In a practical streaming system, such an MBR representation can usually support a small number of bit-rates only, say, $5-8$. However, the reality in the Internet is that the bandwidth can vary among much more rates. To accommodate such a big variation, an efficient solution is to do a fully scalable representation for each source video, such as the fine granularity scalable (FGS) coding scheme developed in MPEG-4 [1] (the layered (scalable) coding scheme developed before MPEG-4 can be treated as a special case of the fully scalable representation). The idea of FGS is to firstly encode an original source video into a coarse base-layer that is very thin so as to fit some small bandwidths. Then, the difference between the original video and the base-layer video forms the enhancement layer and is further encoded using a bit-plane coding technique. Bit-plane coding achieves the desired fine granularity scalability, which offers a fully scalable representation on top of the base-layer. Nevertheless, because of a small bit-rate used at the base-layer, the quality of the coded base-layer video is usually very low. Consequently, the motion compensation 


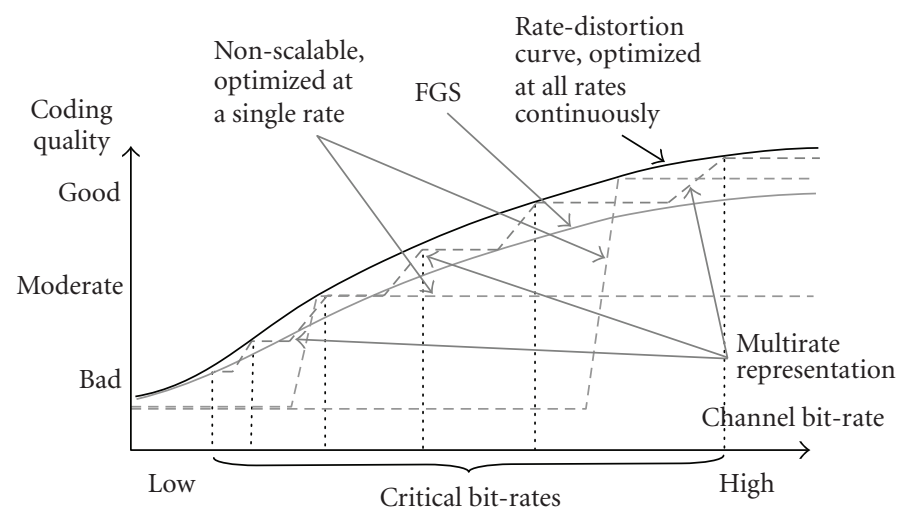

FIgURE 1: Performance comparison of various video coding schemes.

based on the coded base-layer will generally yield a big residual signal, which would cost more bits to represent at the enhancement layer. Experimental results showed that FGS is often $3-5 \mathrm{~dB}$ worse than the corresponding non-scalable coding at the same bit-rate $[2,3]$.

Figure 1 shows conceptually the performances of four coding schemes: the optimal R-D coding (obtained by optimally encoding the source video at every bit-rate continuously), FGS, non-scalable coding (optimized at a single bitrate), and the MBR representation. The goal of designing an MBR representation is to get as close to the R-D curve as possible at each preselected bit-rate, while maintaining a constant performance between two neighboring rates. It can be seen from Figure 1 that the overall performance of an MBR representation could be better than that of the FGS scheme.

In practice, the MBR representation has been adopted in a number of commercial streaming systems such as Windows Media Services, RealSystem, and QuickTime [4-6]. One very striking feature of the MBR method is that not only all source coding tasks but also all channel coding tasks have been completed before the streaming service. As a result, each streaming service is extremely simple: just get the corresponding packets based on the available bandwidth (which determines a bit-rate) and throw them onto networks. On the other hand, a scalable video coding (SVC) scheme (including FGS and the most recent 3D wavelet-based SVC) very likely needs to handle the channel coding (protection, interleaving, packetization, etc.) in a real-time and on-line fashion, which may become a bottleneck problem when a large number of users are served simultaneously.

\subsection{Switching among multiple non-scalable bit-streams}

There are many issues in the MBR representation of a source video, such as how many bit-rates should be used, how to select these critical rates, how to encode a source video at each selected rate (jointly with other rates or independently), and so forth. However, we believe that the most important issue is that an MBR-based streaming system must be equipped with a mechanism that allows effective switching between differ- ent bit-streams when a bandwidth change is detected. In this scenario, let us use $F(t)$ to denote the frame of a video sequence at frame number $t$, and $F_{i}(t)$ to represent the corresponding reconstructed frame at rate $r_{i}(i=1,2, \ldots, M)$. All bits generated after the coding of $F(t)$ at bit-rate $r_{i}$ are grouped into a set $\mathbf{Z}_{i}(t)$, and $C_{i}(t)$ is used to count how many bits are included in this set. Clearly, $C_{i}(t)$ of an intra (I) frame will be much larger than that of a predictive $(\mathrm{P})$ frame because of the motion compensation used in all P-frames. Suppose that a bandwidth change is detected at frame number $t_{0}$ (corresponding to a P-frame) and a switching from $F_{i}(\cdot)$ to $F_{j}(\cdot)$ is needed right at $t_{0}$. The simplest and most straightforward way is to perform the socalled direct switching with the transmitted bit sets being $\left\{\ldots, \mathbf{Z}_{i}\left(t_{0}-1\right), \mathbf{Z}_{j}\left(t_{0}\right), \mathbf{Z}_{j}\left(t_{0}+1\right), \ldots\right\}$. However, since there exists mismatching between $F_{i}\left(t_{0}-1\right)$ and $F_{j}\left(t_{0}-1\right)$, errors will occur when $F_{i}\left(t_{0}-1\right)$ (instead of $F_{j}\left(t_{0}-1\right)$ ) is used to perform the motion compensation for $F_{j}\left(t_{0}\right)$. More seriously, such errors will propagate into all subsequent frames until the next I-frame is received-causing the drifting errors that are often too large to be accepted, especially in the low quality to high quality switching case.

In order to achieve seamless (i.e., no drifting errors) switching, some non-predictive frames can be inserted periodically into each non-scalable bit-stream as key frames, and switching is performed by correctly selecting the nonscalable bit-stream according to the available channel bandwidth and delivering the corresponding key frame to the client [4-6]. To achieve more flexible bandwidth adaptation, more key frame insertion points are needed. However, frequently inserting key frames into a non-scalable bit-stream will seriously degrade the coding efficiency because no temporal correlation is exploited in the coding of a key frame.

Another way to achieve the seamless switching is to transmit the difference between $F_{i}(\cdot)$ and $F_{j}(\cdot)$ at each switching point. Although the temporal redundancy has been exploited in the individual coding of $F_{i}(\cdot)$ or $F_{j}(\cdot)$, lossless representation of the difference between them needs a lot of bits (as overhead) - the number could be much more than that of an I-frame, which is too large to be accepted. As a compromise, further compression is needed to reduce the number 
of overhead bits, while the negative impact is that both $F_{i}(\cdot)$ and $F_{j}(\cdot)$ will be changed at each switching point, thus possibly leading to some quality drop. Furthermore, the coding quality of all subsequent frames before the next I-frame is very likely to drop also.

So far, there have been a few works on how to modify $F_{i}(\cdot)$ and $F_{j}(\cdot)$ so as to achieve the best tradeoff between the number of overhead bits and the quality drop [710]. The so-called SP/SI frames developed for this purpose have been included in the most recent video coding standards H.264/MPEG-4 Part 10 [11] and their R-D performance under various networking conditions has been studied in $[12,13]$. The SP-frame idea has also been applied to achieve seamless switching among scalable bit-streams [14]. One common feature of these works is that the extra processing at each switching point is performed in the DCT coefficient domain. The intrinsic reason lies on the fact that the underlying codec used there is a DCT-based scheme. At this present time, we feel that the compromise achieved so far is still not very satisfactory. For instance, several tens of kilobits are usually needed for each secondary SP-frame of QCIF format and the quality drop is controlled within about $0.5 \mathrm{~dB}$ in the low-to-high switching case [9]. Furthermore, there are many secondary SP-frames at each switching point that need to be generated and stored at server to support arbitrary switching among multiple (more than two) bit-streams.

In our work, we attempt to develop a more effective switching mechanism for multiple non-scalable video bitstreams that can be made seamless at a better R-D performance as compared to those existing schemes. The unique feature of our scheme is that the extra processing at each switching point is performed in a wavelet domain.

The rest of this paper is organized as follows. Section 2 explains how the reconstructed frame $F_{i}(\cdot)$ at each preselected switching point is further processed in the wavelet domain, with emphasis on the optimal bit allocation and the impact on the coding of all subsequent frames. Then, a trivial switching mechanism is presented in Section 3, which is based on independent wavelet processing of the reconstructed frame $F_{i}(\cdot)$ for each rate $r_{i}$. Section 4 presents a joint wavelet processing of two reconstructed frames $F_{i}(\cdot)$ and $F_{j}(\cdot)$ so as to potentially achieve a better rate-distortion performance. Switching among multiple (more than two) bitstreams is studied in Section 5. Some experimental results are given in Section 6. Finally, Section 7 presents the conclusions of this paper.

\section{WAVELET-DOMAIN PROCESSING OF RECONSTRUCTED FRAMES}

To achieve a seamless switching, the reconstructed frame at each switching point need undergo through some extra processing. For instance, such processing is performed in the DCT domain in [7-10, 12-15]. In our work, we propose to perform this extra processing in the wavelet domain. To this end, we apply a wavelet decomposition to the reconstructed frame at each preselected switching points and then perform a lossy coding at a given bit budget. The reason we choose a wavelet coding is twofold: (1) a lot of previous studies proved that the wavelet coding is better than the DCT-based coding; and (2) the wavelet coding can be made scalable easily, which is essential in our MBR-based streaming system to control the overhead budget that is needed at each switching point.

The wavelet coding we have chosen in this paper is the SPIHT algorithm [16]. SPIHT itself is simple and straightforward. The only critical issue here is how to allocate the given bit budget over individual hierarchical trees that are formed after the wavelet decomposition, as discussed in the following.

\subsection{Optimal bit allocation}

The simplest strategy is to average the total budget over all hierarchical trees. However, we know that, due to the spatial location and intrinsic characteristics of individual trees, they play a role with different importance among a whole frame. For example, one can pay more attention to the center of a frame instead of its boundary; while a block that has larger variation tends to be more important toward the overall coding quality. Therefore, a bit allocation optimization is necessary.

Following the SPIHT principle, we know that a number of hierarchical trees, denoted as $T(k), k=1,2, \ldots, K$, are generated after the wavelet decomposition of the reconstructed frame at a switching point. Each tree can be represented into an embedded bit-stream that can be truncated at any position, $n_{k}$. The contribution of $T(k)$ after truncating at $n_{k}$ toward the overall distortion is denoted as $D_{k}\left(n_{k}\right)$. The goal of our optimal bit allocation is to select the truncation position in the embedded bit-stream of each hierarchical tree, that is, $\left\{n_{k} \mid k=1,2, \ldots, K\right\}$, so as to minimize the overall distortion $D=\sum D_{k}\left(n_{k}\right)$ subject to the total budget $B$, that is, $\sum n_{k} \leq B$.

To achieve this goal, one may construct a Lagrangiantype problem and try to solve it. However, since we cannot derive the exact expression of $D_{k}\left(n_{k}\right)$ in terms of $n_{k}$, this problem is not solvable analytically. In our work, we develop the following method: for the $l$ th bit-plane of the $k$ th hierarchical tree, we define a unit coding contribution (UCC) as the ratio of the distortion reduction and the number of bits used to code losslessly the entire bit-plane (using SPIHT), denoted as $S_{l}(k)$.

After computing all $S_{l}(k)$ 's, we rank them from the largest to the smallest. Then, the SPIHT coding always starts with the bit-plane with the largest UCC, continues on the second largest one, and so on. For example, Figure 2(a) shows the coding sequence where 4 hierarchical trees are included and each tree has 3 bit-planes. It is seen from this figure that there are 7 bit-planes totally to be selected/coded for transmission.

However, it is easy to see that such arrangement will run into problem in practice. As the bit-plane $N-1$ of $T_{2}$ is not selected, all bits received for the bit-plane $N-2$ of $T_{2}$ are not decodable. Similarly, as the bit-plane $N-2$ is selected before the bit-plane $N$ in $T_{3}$, all bits in the bit-plane $N-2$ of $T_{3}$ may become undecodable if it happens that some bits in the 


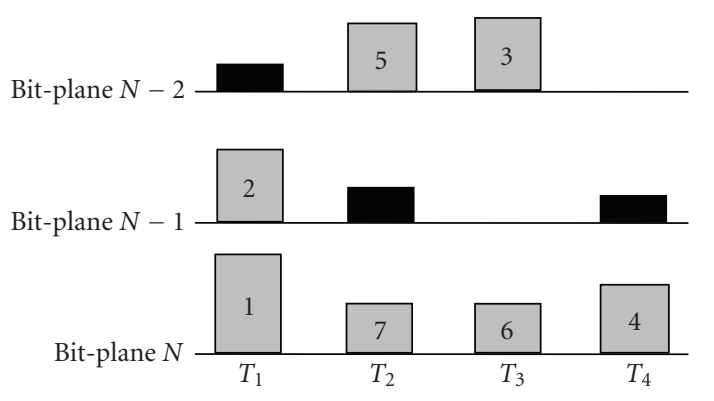

(a)

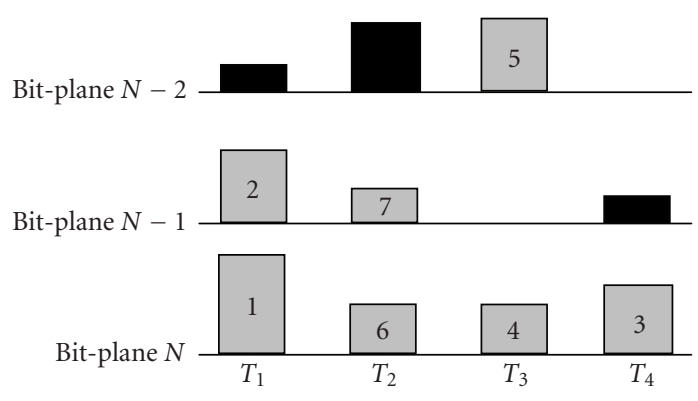

(b)

FIgUre 2: (a) Coding sequence of one example with 4 trees. (b) Adjusted coding sequence of the same example.

bit-plane $N$ of $T_{3}$ are not sent. Some adjustments are therefore necessary. For this example, the correct coding sequence after the adjustment is shown in Figure 2(b).

In practice, we need to compute $S_{l}(k)$, for each rate $r_{i}$, from the reconstructed frame $F_{i}(\cdot)$ at each switching point. Once the coding sequence is determined, we start the SPIHT coding until the given budget $B$ is used up. In this way, $B$ is unevenly allocated over all hierarchical trees. The following matrix shows the actual bit allocation (with the total budget $B=60$ kilobits) for the video sequence "Akiyo" ( $Y$ component) at frame \#15 (the original video sequence of CIF format is coded using H.264 with $Q P=34$ and the 9/7 filter bank is used in the wavelet decomposition of 5 levels): it is seen that the allocation is very uneven:

$$
[\mathrm{BAM}]=[b(u, v)]_{U \times V}=\left[\begin{array}{ccccccccccc}
226 & 216 & 203 & 164 & 43 & 51 & 155 & 171 & 70 & 135 & 342 \\
228 & 286 & 129 & 204 & 798 & 991 & 848 & 305 & 588 & 414 & 398 \\
120 & 256 & 149 & 200 & 881 & 1138 & 835 & 448 & 469 & 352 & 405 \\
87 & 184 & 181 & 158 & 2171 & 1504 & 1225 & 817 & 109 & 187 & 406 \\
256 & 277 & 303 & 187 & 1592 & 1659 & 1191 & 609 & 90 & 171 & 385 \\
129 & 139 & 381 & 834 & 1420 & 1261 & 1306 & 1060 & 674 & 148 & 329 \\
145 & 129 & 1394 & 270 & 614 & 1633 & 1091 & 844 & 453 & 938 & 399 \\
232 & 638 & 588 & 238 & 231 & 1602 & 1156 & 1033 & 330 & 1451 & 307 \\
101 & 770 & 622 & 263 & 163 & 1208 & 3032 & 1340 & 1626 & 941 & 569
\end{array}\right]
$$

with $\sum b(u, v)=B$.

Based on UCC, one bit allocation map $[\mathrm{BAM}]_{i}$ can be derived for each $r_{i}$ at a switching point. It is easy to see that about 1 kilobit ( 12 bits for each element) is needed to losslessly represent this map. It will be seen later on that $[\mathrm{BAM}]_{i}$ may need to be sent (as overhead) during the switching from one bit-stream to another.

\subsection{Influence on coding of subsequent frames}

What is the most important to us is that this SPIHT-based processing of the reconstructed frame at each switching point will unavoidably result in a different frame, and thus may cause some quality drop. More severely, this might influence the coding of all subsequent frames (up to the next I-frame). ${ }^{1}$

\footnotetext{
${ }^{1}$ It is important to notice that the same impact also happens in the SPframe coding scheme in H.264 when comparing against the coding without SP-frames.
}

To understand how big this impact could be, we did many experiments, with some results presented in the following (the original video sequence is coded using H.264 with a QP value specified in each figure).

Figure 3 shows some results where there are 6 frames (one for every 15 frames) specified as switching frames among 100 frames of the "Akiyo," "Foreman," "Stefan," and "Mobile" sequences (all of CIF format and at 30 frames/ second), respectively. At each switching point, the reconstructed frame after the H.264 coding is further processed by SPIHT at $B=53+6+6$ kilobits (for $Y, U$, and $V$ components, resp.) for "Akiyo," $B=70+10+10$ kilobits for "Foreman," $B=140+10+10$ kilobits for "Stefan," and $B=$ $200+15+15$ kilobits for "Mobile," respectively. The optimal bit allocation strategy developed above is used in each SPIHT processing, and the SPIHT processed frames at all switching points are used in the coding of all subsequent frames.

It is seen from these results that all quality curves after performing the SPIHT processing at each switching point 

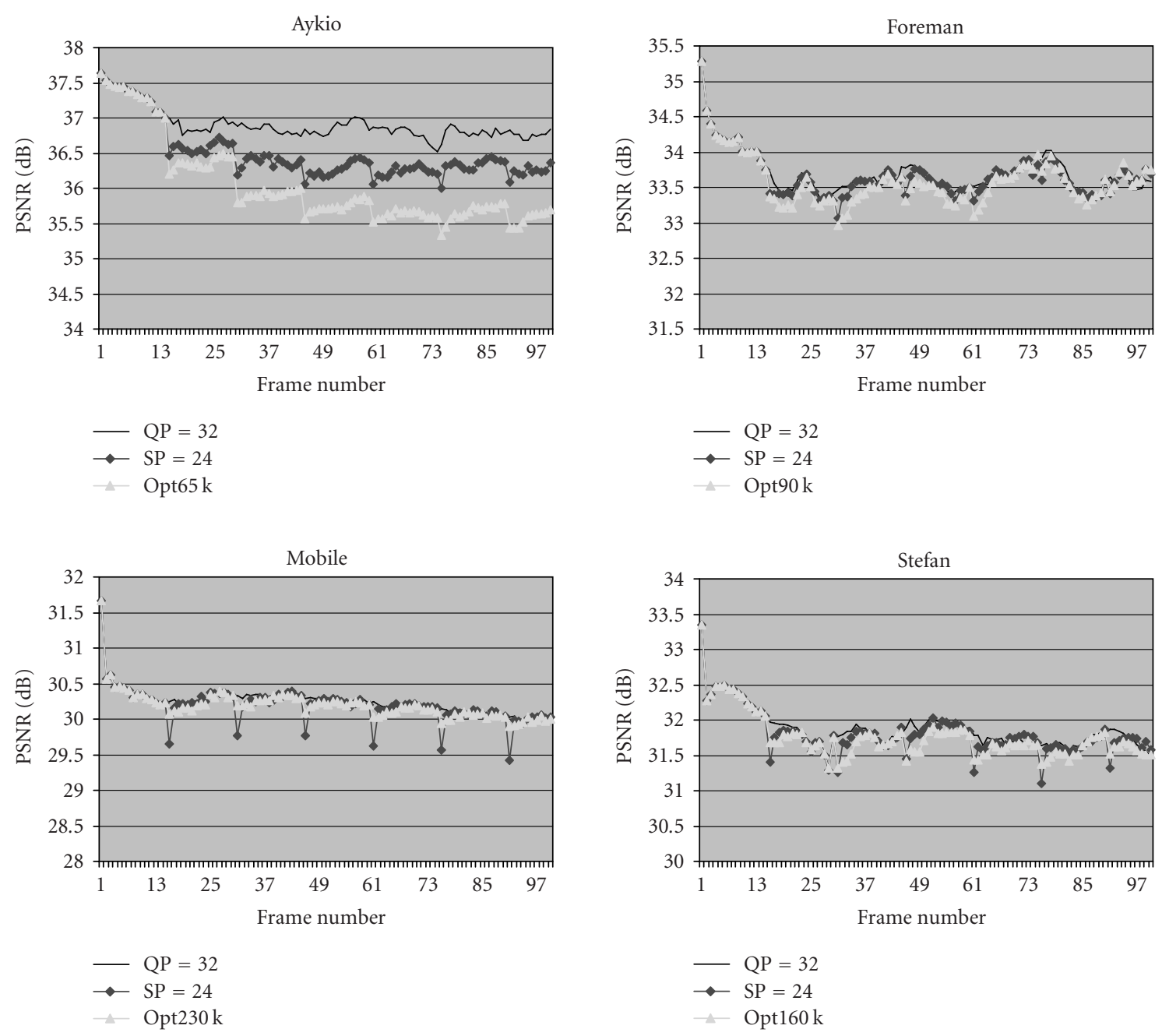

FIgURE 3: Coding quality deviations after six reconstructed frames are further coded using SPIHT.

(the white colored curves with small-triangle markers) do experience certain quality drop, compared to the corresponding curves (the black curves without any markers) where all frames (except for the first one) are coded as P-frames. While the drop in "Akiyo" is quite noticeable (more than $1 \mathrm{~dB}$ ), it is well-controlled within $0.5 \mathrm{~dB}$ for other three sequences. Another interesting observation from Figure 3 is that the coding quality drop at one switching point does not seem to add up with others at all switching points thereafter for "Foreman," "Stefan," and "Mobile," whereas this adding-up effect seems to be existing slightly in "Akiyo."

Figure 3 also includes the corresponding results (the dark grey colored curves with small-diamond markers) obtained by doing a requantization in the DCT domain - the same as was used in H.264 to generate the primary SP-frames [710 ], where the requantization factor SPQP is set at 24. It is clear that the SP-frame scheme yields results that are better than our results for the "Akiyo" sequence, about the same for the "Foreman" sequence, but slightly worse for the "Stefan" and "Mobile" sequences. In the meantime, it is worth to point out that the coding quality drop shown in Figure 3 is much smaller when comparing with what is experienced in the FGS coding (i.e., usually $3 \mathrm{~dB}$ ). A comparison between the bit budget used in the SPIHT processing and the size of each secondary SP-frame generated in H.264 will be presented in the next section.

\section{A TRIVIAL SWITCHING ARRANGEMENT}

After the switching frame $F_{i}\left(t_{0}\right)$ is further processed using the SPIHT algorithm for each rate $r_{i}$ so as to obtain the modified version $\bar{F}_{i}\left(t_{0}\right)$, a trivial switching mechanism between two bit-streams can be arranged as in Figure 4 .

Suppose that the bit-stream at rate $r_{i}$ is currently streamed and a switching to the rate $r_{j}$ is needed right at the preselected point $t_{0}$. Then, the transmitted video frames around the switching point are $\left\{F_{i}\left(t_{0}-1\right), \bar{F}_{j}\left(t_{0}\right), F_{j}\left(t_{0}+\right.\right.$ $1)\}$. From our earlier analysis, we can see that the number of bits used for representing $\bar{F}_{j}\left(t_{0}\right)$ is about 1 kilobit $+B_{j}$, where $B_{j}$ is the total bit budget allowed at each switching 


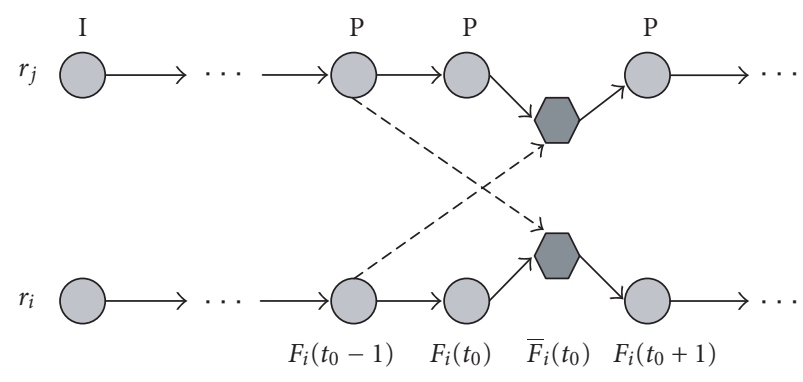

FIGURE 4: A trivial switching arrangement between two bit-streams.

point to SPIHT-code $F_{j}\left(t_{0}\right)$ into $\bar{F}_{j}\left(t_{0}\right)$, and about 1 kilobit is needed to represent $[\mathrm{BAM}]_{j}$ (as $F_{j}\left(t_{0}\right)$ is not available at the switching point-we need to know $[\mathrm{BAM}]_{j}$ so that all received bits for representing $\bar{F}_{j}\left(t_{0}\right)$ can be correctly partitioned among all hierarchical trees). On the other hand, the transmitted frames are $\left\{F_{i}\left(t_{0}-1\right), F_{i}\left(t_{0}\right) / \bar{F}_{i}\left(t_{0}\right), F_{i}\left(t_{0}+1\right)\right\}$ if no switching happens at $t_{0}$. It is important to notice that the SPIHT processing on $F_{i}\left(t_{0}\right)$ so as to generate $\bar{F}_{i}\left(t_{0}\right)$ does not require any extra bits to be sent, because the same processing can be done at the receiver side.

Comparing with the SP-frame switching method in H.264, we see that the frame $\bar{F}_{j}\left(t_{0}\right)$ plays the role of an SPframe at the switching point when a switching from $r_{i}$ to $r_{j}$ indeed happens. Furthermore, it is interesting to notice that $\bar{F}_{j}\left(t_{0}\right)$ also plays the role of an SI-frame for the purpose of splicing and random access/browsing. According to our earlier analysis, the bit count for a switching frame is about 1 kilobit plus the selected budget. For all test sequences used above, we have run H.264 to generate all secondary SPframes under the same configuration as used in Figure 3, and Table 1 presents the sizes of these secondary SP-frames at each preselected switching point for switching between $Q P=28$ and $Q P=36$, with $S P Q P=24$. In fact, we have referred to the bit-counts listed in Table 1 to choose the budget $B$ used above in the SPIHT processing of each switching frame so that $B$ is always significantly $(15 \%-30 \%)$ smaller than the size of the corresponding secondary SP-frame.

In the direct switching scheme, the frames to be transmitted for the switching from $r_{i}$ to $r_{j}$ at the switching point $t_{0}$ is $\left\{F_{i}\left(t_{0}-1\right), F_{j}\left(t_{0}\right), F_{j}\left(t_{0}+1\right)\right\}$. Thus, the bit set $\mathbf{Z}_{j}\left(t_{0}\right)$ needs to be sent right at the switching point $t_{0}$. In most coding applications, $C_{j}\left(t_{0}\right)$-the bit count in $\mathbf{Z}_{j}\left(t_{0}\right)$ would be much smaller than $B_{j}$. For instance, the typical value of $C_{j}\left(t_{0}\right)$ is about 2-4 kilobits in the coding of video sources of 30 frames/second at 128 kilobits/second, whereas $B_{j}$ is usually several tens of kilobits.

Another feature of this trivial switching arrangement is that two reconstructed frames are independently processed (using SPIHT) according to their individual budgets. In reality, however, we know that there typically exists a strong similarity between these two reconstructed frames so that a joint processing seems more appropriate. Such a joint processing will be presented in the next section.

\section{JOINT PROCESSING OF SWITCHING FRAMES AT TWO BIT-RATES}

We only consider the switching between two non-scalable bit-streams in this section, while the extension to multiple (more than two) bit-streams is discussed later in Section 5. In this scenario, we feed both reconstructed frames at each preselected switching point into a joint SPIHT-type processing, as shown in Figure 5.

The upper part outlined by the dash-line box is the nonscalable coding of a source video at the higher bit-rate $r_{H}$, and the corresponding coding at the lower bit-rate $r_{L}$ is shown in the bottom part. After the reconstruction, however, two coded versions at bit-rates $r_{H}$ and $r_{L}$ are fed into the joint SPIHT box for some extra processing, as outlined in the following.

Step 1. Both $F_{H}\left(t_{0}\right)$ and $F_{L}\left(t_{0}\right)$ at a preselected switching point $t_{0}$ undergo the same wavelet decomposition with the maximum depth (e.g., 5 levels are needed in the CIF format) to generate all hierarchical trees $T_{H}(u, v)$ and $T_{L}(u, v)$ (e.g., there are totally $9 \times 11=99$ hierarchical trees in the CIF format).

Step 2. The SPIHT coding is performed on $F_{H}\left(t_{0}\right)$ and $F_{L}\left(t_{0}\right)$, respectively, according to their bit allocation maps $[\mathrm{BAM}]_{H}$ and $[\mathrm{BAM}]_{L}$ that can be derived from the allowed total budgets $B_{H}$ and $B_{L}$. After the SPIHT coding, each hierarchical tree is denoted as $\bar{T}_{H}(u, v)$ or $\bar{T}_{L}(u, v)$, with length $b_{H}(u, v)$ or $b_{L}(u, v)$, respectively.

Step 3. We start a joint processing on two coded hierarchical trees $\bar{T}_{H}(u, v)$ and $\bar{T}_{L}(u, v)$ (for each $(u, v)$ ) by representing the difference between them.

Some explanations are in order. First of all, the coding of all subsequent frames after a switching point $t_{0}$ is based on the modified versions of $F_{H}\left(t_{0}\right)$ and $F_{L}\left(t_{0}\right)$, that is, $\bar{F}_{H}\left(t_{0}\right)$ and $\bar{F}_{L}\left(t_{0}\right)$, as shown in Figure 5, no matter whether a switching indeed happens or not at $t_{0}$ during streaming. This usually will cause some quality drop. From our study presented in Section 2, such quality drop has been controlled within a small level. Secondly, when no switching happens at time $t_{0}$, the frame $F_{H}\left(t_{0}\right)$ or $F_{L}\left(t_{0}\right)$ reconstructed at the decoder side has to undergo the same (as what is done at the encoder side) wavelet compression so as to generate $\bar{F}_{H}\left(t_{0}\right)$ or $\bar{F}_{L}\left(t_{0}\right)$ (for synchronizing the encoder and the decoder). In practice, this is doable as we know the budget $B_{H}$ or $B_{L}$ at the decoder side so that the same $[\mathrm{BAM}]_{H}$ or $[\mathrm{BAM}]_{L}$ can be derived. Thus, zero overhead bits are needed if no switching happens at $t_{0}$. Thirdly, all bits representing the difference between $\bar{F}_{H}\left(t_{0}\right)$ and $\bar{F}_{L}\left(t_{0}\right)$ need to be sent as overhead when a switching between $r_{L}$ and $r_{H}$ does happen at $t_{0}$.

The block diagram of representing the difference between $\bar{F}_{H}\left(t_{0}\right)$ and $\bar{F}_{L}\left(t_{0}\right)$ (during the SPIHT coding process of individual hierarchical trees) is as simple as shown in Figure 6, with principle as follows: (1) a bit 0 is recorded if the first corresponding bits of $\bar{T}_{H}(u, v)$ and $\bar{T}_{L}(u, v)$ are the same, and we continuously record the bit 0 if the following corresponding 
TABLE 1: Bit counts of the secondary SP frames in various test sequences.

\begin{tabular}{lccccccc}
\hline Sequence & Switching direction & Switching \#1 & Switching \#2 & Switching \#3 & Switching \#4 & Switching \#5 & Switching \#6 \\
\hline \multirow{2}{*}{ Akiyo } & QP: $28 \rightarrow 36$ & 72,600 & 73,688 & 73,656 & 73,336 & 74,848 \\
& QP: $36 \rightarrow 28$ & 74,376 & 75,440 & 75,384 & 75,152 & 76,696 & 75,216 \\
\hline \multirow{2}{*}{ Foreman } & QP: $28 \rightarrow 36$ & 112,600 & 111,240 & 107,496 & 113,408 & 107,2484 \\
& QP: $36 \rightarrow 28$ & 117,448 & 115,600 & 111,632 & 117,872 & 111,776 & 123,304 \\
\hline \multirow{2}{*}{ Stefan } & QP: $28 \rightarrow 36$ & 199,288 & 196,504 & 199,240 & 194,160 & 194,520 & 203,424 \\
& QP: $36 \rightarrow 28$ & 207,176 & 203,688 & 208,816 & 205,504 & 207,120 & 216,504 \\
\hline \multirow{2}{*}{ Mobile } & QP: $28 \rightarrow 36$ & 254,584 & 251,600 & 254,784 & 257,600 & 265,888 & 272,720 \\
& QP: $36 \rightarrow 28$ & 275,456 & 272,768 & 274,432 & 280,352 & 288,888 & 296,200 \\
\hline
\end{tabular}

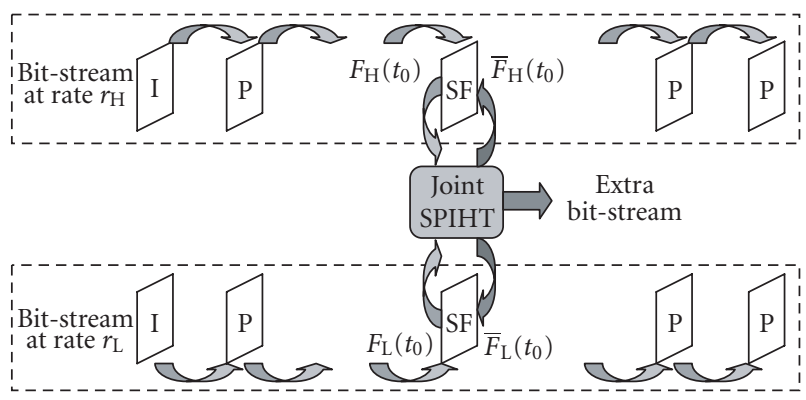

FIGURE 5: Joint SPIHT processing of two reconstructed frames at each switching point.

bits of $\bar{T}_{H}(u, v)$ and $\bar{T}_{L}(u, v)$ are also the same (e.g., the first 5 bits of $\bar{T}_{H}(u, v)$ and $\bar{T}_{L}(u, v)$, shown by the concatenated squares in Figure 6); and (2) as long as we observe that the corresponding bits of $\bar{T}_{H}(u, v)$ and $\bar{T}_{L}(u, v)$ are not the same for the first time, all remaining bits of $\bar{T}_{H}(u, v)$ (the white colored horizontal bar shown in Figure 6) are recorded into the box denoted as "extra bit-stream for switching up" (i.e., from $r_{L}$ to $r_{H}$ ); while all remaining bits of $\bar{T}_{L}(u, v)$ (the gray colored horizontal bar shown in Figure 6) are recorded into the box denoted as "extra bit-stream for switching down" (i.e., from $r_{H}$ to $r_{L}$ ).

Because both $F_{L}\left(t_{0}\right)$ and $F_{H}\left(t_{0}\right)$ are coded from the same original frame $F\left(t_{0}\right)$, there exists a high degree of similarity between them. Thus, a lot of leading bits in the coding of two corresponding hierarchical trees $T_{H}(u, v)$ and $T_{L}(u, v)$ would be the same for each $(u, v)$. In practice, instead of sending these leading 0 bits (as deleted by a big cross in Figure 6), we use an integer $N(u, v)$ to represent the runlength so as to achieve a much higher efficiency. In our simulations, we observed that the number of these same leading bits is often quite large, with the maximum and average being about 250 and 60 , respectively, which thus can be fully covered by 8 bits.

No matter a switching between $r_{L}$ and $r_{H}$ indeed happens or not at $t_{0}$ during the practical streaming service, we always send the bit set $\mathbf{Z}_{L}\left(t_{0}\right)$ or $\mathbf{Z}_{H}\left(t_{0}\right)$ (needing $C_{L}\left(t_{0}\right)$ or $C_{H}\left(t_{0}\right)$ bits, resp.) so that we know either $F_{L}\left(t_{0}\right)$ or $F_{H}\left(t_{0}\right)$ at this switching point. Obviously, zero overhead bits are needed if no switching happens at $t_{0}$. However, we need to

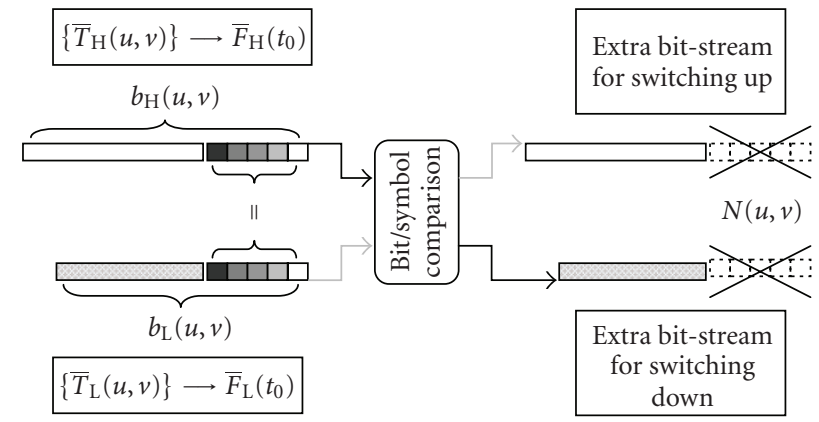

FIGURE 6: Block diagram for the joint SPIHT processing of two coded frames at a switching point.

use the reconstructed $F_{L}\left(t_{0}\right)$ or $F_{H}\left(t_{0}\right)$ to compute the bit allocation map $[\mathrm{BAM}]_{L}$ or $[\mathrm{BAM}]_{H}$ according to the given total budget $B_{L}$ or $B_{H}$ (as discussed in Section 2), and then $F_{L}\left(t_{0}\right)$ or $F_{H}\left(t_{0}\right)$ needs to go through the SPIHT processing so as to generate $\bar{F}_{L}\left(t_{0}\right)$ or $\bar{F}_{H}\left(t_{0}\right)$.

On the other hand, if a switching does happen at $t_{0}$, we still can compute one bit allocation map $[\mathrm{BAM}]_{L}$ or $[\mathrm{BAM}]_{H}$ (as either $F_{L}\left(t_{0}\right)$ or $F_{H}\left(t_{0}\right)$ is also available), while the other map needs about 1 kilobit (as overhead) to represent. ${ }^{2}$ Then, $F_{L}\left(t_{0}\right)$ or $F_{H}\left(t_{0}\right)$ goes through the SPIHT processing according to the computed bit allocation map. However, we only keep the first $N(u, v)$ bits during the SPIHT coding of its $(u, v)$ th hierarchical tree. According to our earlier discussion, these first $N(u, v)$ bits are the same in the coding of the $(u, v)$ th hierarchical tree of both $F_{L}\left(t_{0}\right)$ and $F_{H}\left(t_{0}\right)$, while $N(u, v)$ itself needs 8 bits to represent. Therefore, we can derive that the total number of bits to be sent for a switching is

$$
\begin{aligned}
\mathfrak{R}_{H \mapsto L(\text { or } L \hookleftarrow H)}= & E+B_{L(\text { or } H)}+C_{H(\text { or } L)}\left(t_{0}\right) \\
& +8 \cdot(U \times V)-\sum N(u, v),
\end{aligned}
$$

where $E$ denotes the number of bits used to represent the dif-

\footnotetext{
2 Alternatively, we can represent the difference between these two maps so as to reduce the overhead bit count, and this strategy has been adopted in our system.
} 


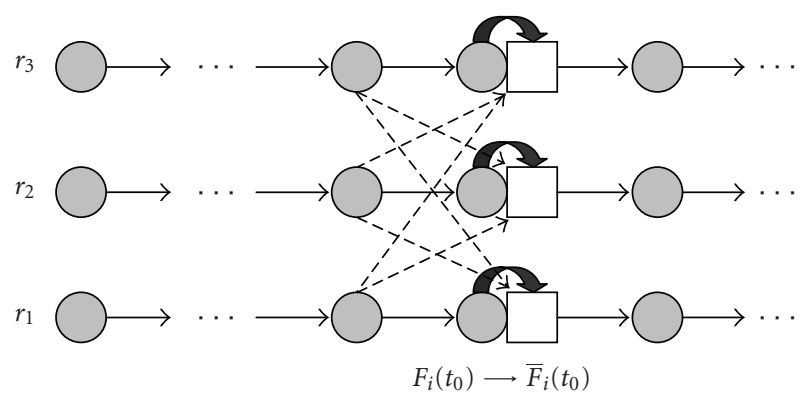

Figure 7: Switching among three bit-streams at the preselected point.

ference between $[\mathrm{BAM}]_{L}$ and $[\mathrm{BAM}]_{H}$ (which is now smaller than 1 kilobit for the CIF format), and $U=9$ and $V=11$ for the CIF format. It is clear that this new switching arrangement becomes more efficient than the trivial switching mechanism presented in Section 3 as long as $\sum N(u, v)>$ $E+C_{H(\text { or } L)}\left(t_{0}\right)+8 \cdot(U \times V)-1$ kilobit.

\section{SWITCHING AMONG MULTIPLE BIT-STREAMS}

For switching among more than two bit-streams coded at rates $r_{i}, i=1,2, \ldots, M$, each reconstructed frame $F_{i}\left(t_{0}\right)$ at the switching point $t_{0}$ is coded using SPIHT at the selected budget $B_{i}$ so as to generate $\bar{F}_{i}\left(t_{0}\right)$. Then, the trivial switching arrangement developed in Section 3 can be extended readily to this multiple bit-rate case, see Figure 7 for an example where $M=3$ and only one switching point is included. Clearly, this arrangement allows any arbitrary switching, that is, between rate $r_{i}$ and rate $r_{j}$ for all $j \neq i$. As discussed in Section 3, the total number of bits to be sent is about 1 kilobit $+B_{i}$ if a switching from any rate to $r_{i}$ indeed happens at a preselected switching point $t_{0}$. As discussed in Section 4, this number could be further reduced by using the joint SPIHT processing between $r_{i}$ and $r_{j}$. Therefore, the joint processing will be enforced at a switching point only when it can reduce the count of overhead bits that needs to be sent. On the other hand, no overhead bits are sent if no switching happens at $t_{0}$ : only the bit set $\mathbf{Z}_{i}\left(t_{0}\right)$ needs to be sent, whereas the corresponding SPIHT needs to be performed at the decoder side so as to generate $\bar{F}_{i}\left(t_{0}\right)$.

It is clear from Figure 7 that we need to store a number of $M$ extra frames $\bar{F}_{i}\left(t_{0}\right), i=1,2, \ldots, M$, at the video server, to support any arbitrary switching between $r_{i}$ and $r_{j}$ for all $j \neq$ $i$. On the other hand, there are totally $M \cdot(M-1)$ secondary SP-frames that need to be generated and stored at the server in the SP-frame switching scheme to support any arbitrary switching - which is obviously very disadvantageous.

Compared to the scheme proposed in [15] where a new bit-stream (called the S-stream) is generated at each switching point and it will be selected when a switching indeed happens at this point, each switching frame in our method is generated in the intra-coding manner. As a result, each switching frame generated in our method serves both the switching task and the purpose of random access and browsing. Furthermore, it has been demonstrated in [9] that each $\mathrm{S}$-stream is less efficient than the corresponding SP-frame switching, whereas some results will be presented in the next section to show that our switching scheme provides a better rate-distortion performance than the SP-frame switching.

In principle, we should select different bit budget $B_{i}$ for different rate $r_{i}$ in the implementation of our switching scheme. In reality, however, it is rather difficult to establish an accurate relationship between them. For instance, it is not necessarily true that a smaller budget should be used for a smaller rate. In our H.264-based experiments, we observed that, in the switching-down case, the size of the secondary SP-frame for switching from the maximum rate to the minimum rate is actually larger than that of the corresponding secondary SP-frame for switching from the same maximum rate to any of other lower rates (not the minimum one). This result seems to be rather absurd: more overhead bits need to be sent when a bigger bandwidth drop is detected! In fact, how to handle this problem is left over as one of our future works.

For simplicity, we choose the budget $B_{i}$ for each rate based on the sizes of the corresponding secondary SP-frames. For example, for $r_{i}$, there are $M-1$ switching-in cases (from $r_{j}$ for all $j \neq i$ ) at each switching point. Then, we run H.264 with a selected SPQP to obtain the sizes of all $M-1 \mathrm{sec}-$ ondary SP-frames, and choose $B_{i}$ at a number that is slightly smaller than the minimum size. In general, for each $r_{i}$, this will result in different $B_{i}$ at different switching points. In our simulations, however, we try to ignore this variation and thus use the same $B_{i}$ at all switching points.

\section{EXPERIMENTAL RESULTS AND ANALYSIS}

In this section, we provide some experimental results to illustrate the coding efficiency of our proposed switching method. In our simulations, 5 bit-streams are generated by using H.264 at different QP factors: $Q P 5=24, Q P 4=28$, $Q P 3=32, Q P 2=36$, and $Q P 1=40$, respectively. Overall, 100 frames are encoded, with the first frame as I-frame and the rest of them as P-frames. Then, six switching points are selected at $\# 15, \# 30, \# 45, \# 60, \# 75$, and $\# 90$, respectively. The switching arrangement is similar to the one shown in Figure 7 , while the $9 / 7$ filter bank is used to perform the wavelet decomposition of 5 levels.

Figure 8 shows the results in terms of luminance PSNR for the "Foreman" and "Stefan" sequences, while Table 2 lists the bit budgets used to obtain these results in which 20 kilobits are used for the $U$ and $V$ components and the remaining is for the $Y$ component. It should be pointed out that these budgets are determined by referring to the sizes of the corresponding secondary SP-frames obtained in running H.264 with a fixed SPQP at 24 for all rates (see the discussions at the end of Section 5). Thus, different budgets may be used if other SPQP values are used to generate SP-frames.

For each of these two sequences, the first plot shows the monotonic switching-up scenario, the second one shows the 

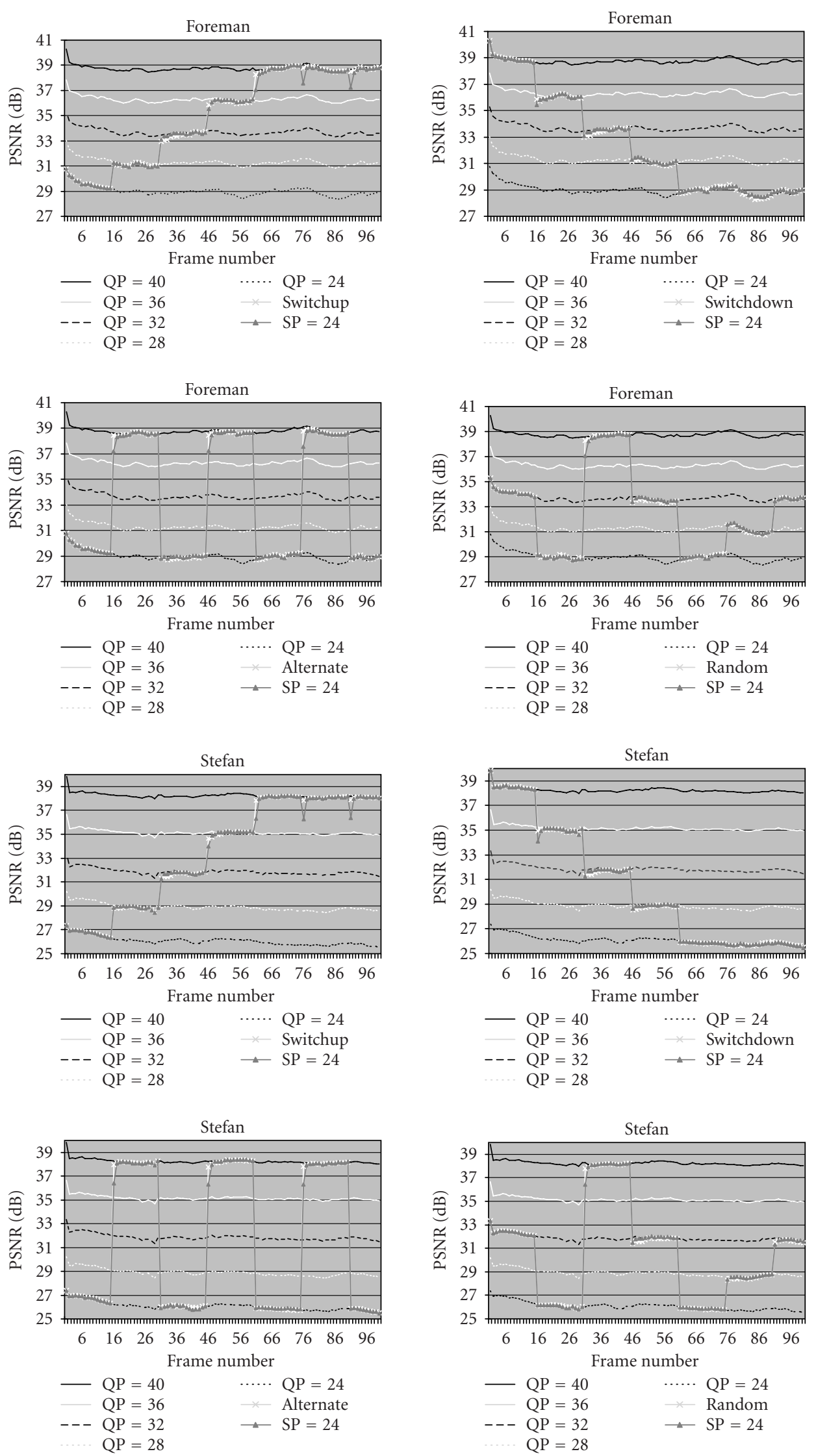

FIGURE 8: Four switching scenarios among five bit-streams of "Foreman" and "Stefan." 
TABLE 2: Budgets (in kilobits) used in our simulations—same at all switching points.

\begin{tabular}{lccccc}
\hline Sequence & $Q P=40$ & $Q P=36$ & $Q P=32$ & $Q P=28$ & $Q P=24$ \\
\hline Foreman & $75+10+10$ & $75+10+10$ & $70+10+10$ & $60+10+10$ & $65+10+10$ \\
Stefan & $170+10+10$ & $160+10+10$ & $120+10+10$ & $120+10+10$ & $130+10+10$ \\
\hline
\end{tabular}

TABLE 3: Sizes of secondary SP-frames to be sent at each switching point (in bits).

\begin{tabular}{lccccccc}
\hline Sequence & Scenario & $\# 1$ & $\# 2$ & $\# 3$ & $\# 4$ & $\# 5$ & $-\#$ \\
\hline \multirow{3}{*}{ Foreman } & Up & 109,792 & 101,880 & 97,664 & 96,496 & - \\
& Down & 95,584 & 96,920 & 98,672 & 105,992 & - \\
& Alternate & 142,504 & 136,968 & 139,120 & 137,688 & 137,680 \\
& Random & 116,896 & 144,472 & 103,904 & 117,352 & 103,552 & 110,256 \\
\hline \multirow{3}{*}{ Stefan } & Up & 202,272 & 185,920 & 177,936 & 156,176 & - \\
& Down & 159,504 & 169,104 & 185,688 & 195,208 & - \\
& Alternate & 239,224 & 233,576 & 251,232 & 228,912 & 253,576 & 237,504 \\
& Random & 207,408 & 244,616 & 177,800 & 209,608 & 198,024 & 200,424 \\
\hline
\end{tabular}

monotonic switching-down scenario, the third one shows the alternate switching scenario between the minimum rate and the maximum rate, and the fourth one shows a scenario of random switching (both up and down). Five black or white curves without markers in Figure 8 represent the H.264-coded results with all frames (except for the first one) coded as P-frames. Therefore, it is expected that the quality curve after inserting some switching points will always be (slightly) worse. However, it is seen from Figure 8 that the results achieved in our switching scheme (the white curves with small cross markers) are nearly perfect at all switching points for both sequences.

Figure 8 also presents the results obtained by using the SP-frame switching scheme (the dark grey curves with small triangle markers), and Table 3 summarizes the sizes of the corresponding secondary SP-frames that need to be sent at each switching point. It is seen that while the resulting quality curves are nearly the same as our results, the SP-frame switching scheme requires many more bits to be sent at each switching point.

\section{CONCLUSIONS AND FUTURE WORKS}

Multirate representation seems to be one efficient solution to the video streaming service over heterogeneous and dynamic networks. In this paper, we developed an effective method that allows seamless switching among different bit-streams in multirate based streaming systems when a channel bandwidth change is detected. The unique feature of our method is that, at a preselected switching point, the reconstructed frame at each rate or two reconstructed frames at different rates need undergo through an independent or a joint SPIHT-type processing in the wavelet domain in which an optimal bit allocation over all hierarchical trees has been applied. Compared with the SP-frame switching scheme, our method proves to be able to achieve the seamless switching at a better rate-distortion performance.
Our future works will be focusing on how to handle the switching-down case more effectively so that much fewer bits need to be sent in this scenario. On the other hand, we know that the SPIHT coding plays a critical role in our switching scheme. Although SPIHT itself is quite efficient, trying to further increase the coding efficiency is also one of our future works. In the meantime, we will also consider other popular ways to accommodate possible bandwidth changes, such as frame-skipping and down-sizing, so as to facilitate a more practical streaming system.

\section{ACKNOWLEDGMENTS}

This work has been supported partly by a DAG research grant from HKUST and a RGC research grant from HKSAR. We would like to thank Dr. Xiaoyan Sun of Microsoft Research Asia for helping us get the bit-counts listed in Tables 1, 2, and 3 .

\section{REFERENCES}

[1] ISO/IEC 14496-2, "Coding of audio-visual objects, part-2: visual," December 1998.

[2] W. Li, "Overview of fine granularity scalability in MPEG-4 video standard," IEEE Transactions on Circuits and Systems for Video Technology, vol. 11, no. 3, pp. 301-317, 2001.

[3] F. Wu, S. Li, and Y.-Q. Zhang, "A framework for efficient progressive fine granularity scalable video coding," IEEE Transactions on Circuits and Systems for Video Technology, vol. 11, no. 3, pp. 332-344, 2001.

[4] D. Wu, Y. T. Hou, W. Zhu, Y.-Q. Zhang, and J. M. Peha, "Streaming video over the internet: approaches and directions," IEEE Transactions on Circuits and Systems for Video Technology, vol. 11, no. 3, pp. 282-300, 2001.

[5] G. J. Conklin, G. S. Greenbaum, K. O. Lillevold, A. F. Lippman, and Y. A. Reznik, "Video coding for streaming media delivery on the internet," IEEE Transactions on Circuits and Systems for Video Technology, vol. 11, no. 3, pp. 269-281, 2001. 
[6] J. Lu, "Signal processing for internet video streaming: a review," in Image and Video Communications and Processing, vol. 3974 of Proceedings of SPIE, pp. 246-259, San Jose, Calif, USA, January 2000.

[7] M. Karczewicz and R. Kurceren, "A proposal for SP-frames," in ITU-T Video Coding Experts Group Meeting, Eibsee, Germany, January 2001, Doc. VCEG-L-27.

[8] M. Karczewicz and R. Kurceren, "Improved SP-frame encoding," in ITU-T Video Coding Experts Group Meeting, Austin, Tex, USA, April 2001, Doc. VCEG-M-73.

[9] M. Karczewicz and R. Kurceren, "The SP- and SI-frames design for H.264/AVC," IEEE Transactions on Circuits and Systems for Video Technology, vol. 13, no. 7, pp. 637-644, 2003.

[10] X. Sun, S. Li, F. Wu, G. Shen, and W. Gao, "Efficient and flexible drift-free video bitstream switching at predictive frames," in Proceedings of IEEE International Conference on Multimedia and Expo (ICME '02), Lausanne, Switzerland, August 2002.

[11] ITU-T Rec. H.264 | ISO/IEC 14496-10 (AVC), "Advanced video coding for generic audiovisual services”, March 2005.

[12] E. Setton and B. Girod, "Video streaming with SP and SI frames," in Visual Communications and Image Processing (VCIP '05), vol. 5960 of Proceedings of SPIE, pp. 2204-2211, Beijing, China, July 2005.

[13] E. Setton, P. Ramanathan, and B. Girod, "Rate-distortion analysis of SP and SI frames," in Proceedings of IEEE International Conference on Image Processing (ICIP '05), Genova, Italy, September 2005.

[14] X. Sun, F. Wu, S. Li, W. Gao, and Y.-Q. Zhang, "Seamless switching of scalable video bitstreams for efficient streaming," IEEE Transactions on Multimedia, vol. 6, no. 2, pp. 291-303, 2004.

[15] N. Farber and B. Girod, "Robutst H.263 compatible video transmission for mobile access to video servers," in Proceedings of IEEE International Conference on Image Processing (ICIP '97), Santa Barbara, Calif, USA, October 1997.

[16] A. A. Said and W. A. Pearlman, "New, fast, and efficient image codec based on set partitioning in hierarchical trees," IEEE Transactions on Circuits and Systems for Video Technology, vol. 6, no. 3, pp. 243-250, 1996.

Wei Zhang received the B.Eng. and M.Phil. degrees in electronic engineering from Hong Kong University of Science and Technology, Hong Kong, in 2003 and 2006, respectively. Her research interests include video/image coding and video streaming.

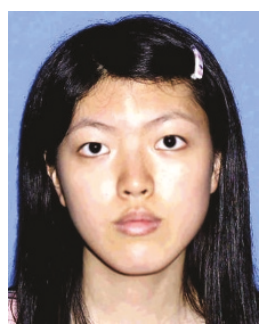

Bing Zeng joined the Hong Kong University of Science and Technology in 1993 and is currently an Associate Professor at the Department of Electrical and Electronic Engineering. His general research interests include digital signal and image processing, linear and nonlinear filter design, and image/video coding and transmission. His most recent research focus is on some fundamental issues in image/video coding such as directional transform, truly optimal rate allocation, and smart motion estimation/compensation, as well as various solutions for real-time video streaming applications over the Internet and

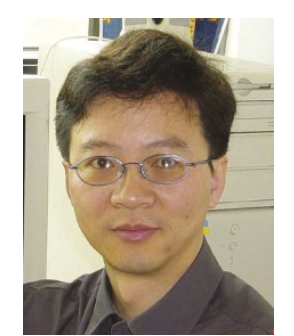

wireless networks. His research efforts in these areas have produced over 150 journal and conference publications. He received the B.Eng. and M.Eng. degrees from the University of Electronic Science and Technology of China in 1983 and 1986, respectively, and the Ph.D. degree from Tampere University of Technology, Finland, in 1991, all in electrical engineering. He worked as a Postdoctoral Fellow at the University of Toronto and Concordia University during 1991-1993 and was a Visiting Researcher at Microsoft Research Asia, Beijing, China, in 2000. He was an Associate Editor for the IEEE Transactions on Circuits and Systems for Video Technology during 1995-1999 and served in various capacities in a number of international conferences. He is currently a Member of the Visual Signal Processing \& Communications Technical Committee of IEEE CAS Society. 